\title{
Compressed Sensing Electron Tomography: Theory and Applications
}

\author{
Rowan Leary ${ }^{1}$, Zineb Saghi ${ }^{1}$, Paul A. Midgley ${ }^{1}$ and Daniel J. Holland ${ }^{2}$ \\ 1. Department of Materials Science and Metallurgy, University of Cambridge, Pembroke Street, \\ Cambridge, CB2 3QZ, UK. \\ 2. Department of Chemical Engineering and Biotechnology, University of Cambridge, New Museums \\ Site, Pembroke Street, Cambridge CB2 3RA, UK.
}

Electron tomography (ET) is now a widely used technique for three-dimensional (3D) nanoscale characterization in the physical and life sciences. An angular series of projections (2D images) is recorded using a transmission electron microscope, typically about a single tilt axis, and established algorithms are used to reconstruct the 3D morphology, composition or other physical properties of nanoscale specimens. However, ET studies frequently suffer from significant artifacts arising from the limited tilt range that is accessible and the restricted number of projections that can be recorded due to the electron beam sensitivity of the sample. The development of sophisticated reconstruction algorithms, capable of providing high-fidelity 3D reconstructions from such limited data is therefore of great importance, particularly if quantitative ET studies are to be undertaken.

The recent mathematical concept of compressed sensing (CS) [1,2] asserts that a small number of wellchosen measurements can suffice to reconstruct signals that are amenable to sparse or compressible representation. In addition to powerful theoretical results, the principles of CS are being exploited across a range of experiments to yield substantial performance gains relative to conventional approaches. Here we describe the salient aspects of CS theory and the application of CS to ET reconstruction, and demonstrate the efficacy of CS-ET with several example studies involving both simulated and experimental data.

The key prior knowledge harnessed in CS is that the signal is sparse or compressible in some known transform domain, meaning that it can be represented or well-approximated, respectively, in a more compact form. A wide variety of transforms are available for this task, making the applicability of CS wide-reaching. Implicit sparsity is indeed prevalent at the nanoscale, and we have exploited a number of effective sparsifying transforms in the application of CS-ET to different specimens. CS reconstruction is accomplished using a non-linear algorithm that promotes sparsity by minimizing the number of non-zero coefficients in the sparse domain, whilst also ensuring consistency with the measured data (Figure 1). Using CS-ET, artifacts present in conventional ET reconstructions such as streaking, blurring of object boundaries and elongation are markedly reduced, and robust reconstruction is possible from far fewer projections than are normally used. Importantly, the CS-ET reconstructions can be more reliably segmented and analyzed quantitatively (Figure 2).

CS-ET opens the door to novel ET studies in a number of important areas, including 3D mapping of physical properties [3] and atomic scale analysis [4], and there is clear potential for the study of electron beam sensitive specimens, as well as use in conjunction with in-situ and time-resolved techniques. Moreover, the reconstruction capabilities suggest a reconsideration of acquisition protocols in general.

References

[1] EJ Candès, J Romberg and T Tao, IEEE Trans. Inf. Theory 52 (2006) p. 489.

[2] DL Donoho, IEEE Trans. Inf. Theory 52 (2006) p. 1289. 
[3] O Nicoletti et al, (submitted).

[4] B Goris et al, Nat. Mater. 11 (2012) p. 930.

[5] The research leading to these results has received funding from the European Research Council under the European Union's Seventh Framework Programme (FP7/2007-2013)/ERC grant agreement 291522-3DIMAGE. DJH acknowledges the support of the EPSRC (EP/F041772/1 and EP/F047991/1).
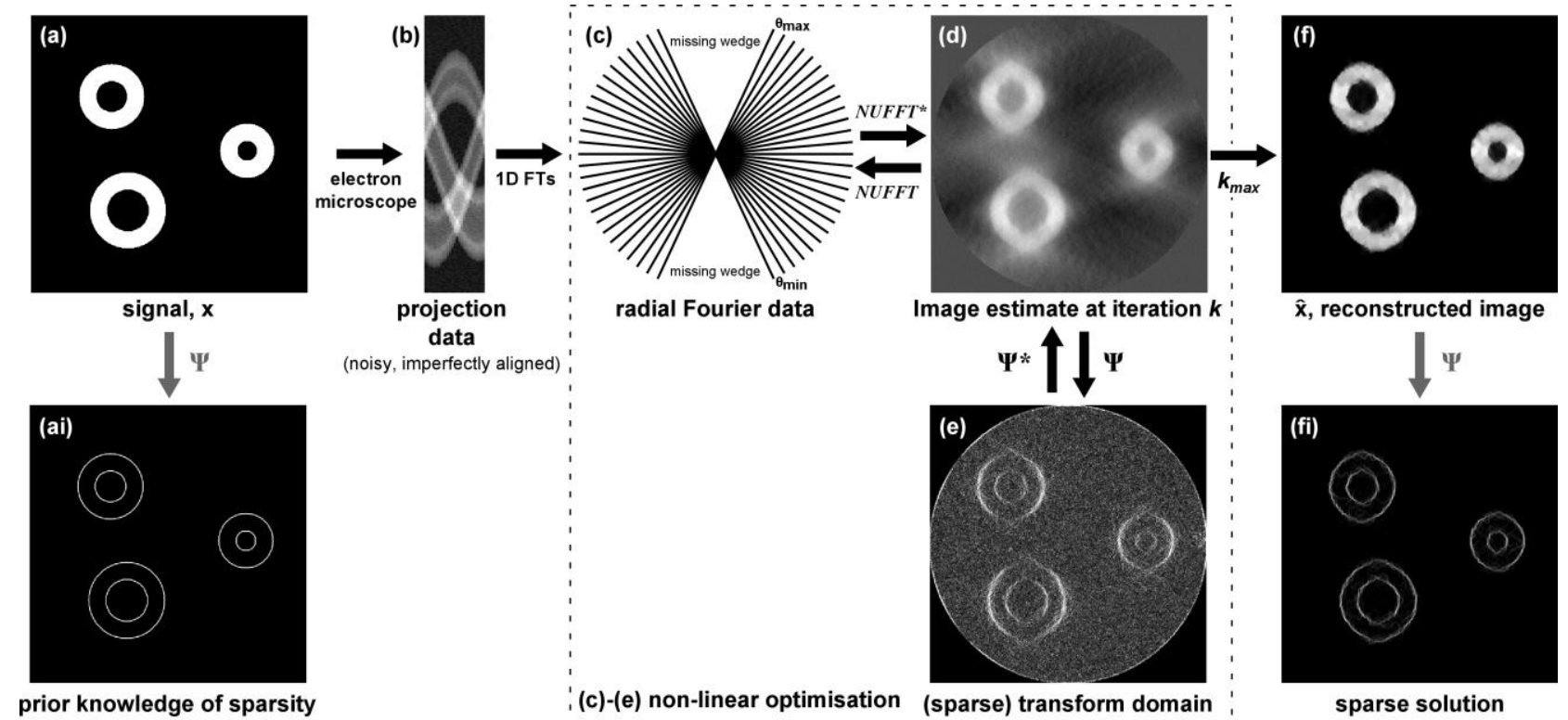

prior knowledge of sparsity

( (c)-(e) non-linear optimisation

(sparse) transform domain

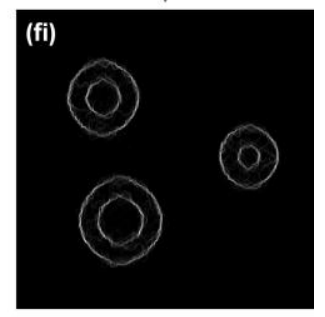

sparse solution

Figure 1. Overview of CS-ET. (a) CS requires that it be possible to represent the signal sparsely using an appropriate transform, $\Psi$. In the CS framework, linear non-adaptive global measurements are made against some test function; in ET a limited number of projection images (b) are acquired in the (S)TEM over a finite tilt range. CS recovery proceeds via a sparsity promoting non-linear optimization process (c-e), minimizing the number of non-zero coefficients in the (sparse) transform domain (e), whilst ensuring consistency with the measured data (c) (shown here following a Fourier based approach for tomographic reconstruction). Incoherent undersampling results in artifacts that appear noise-like and distributed throughout the transform domain (e). These artifacts are suppressed during CS reconstruction, enabling the larger coefficients corresponding to the signal to be recovered accurately.
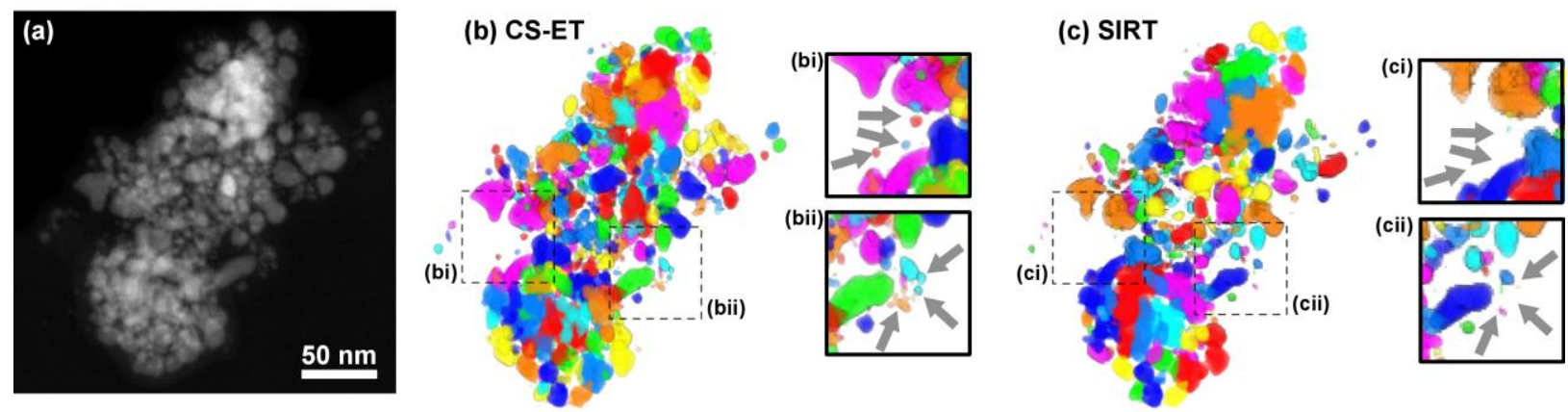

Figure 2. (a) HAADF-STEM image of Ga-Pd nanoparticles from an ET tilt series. (b,c) 3D voxel projection visualizations of segmented CS-ET and SIRT reconstructions, obtained by global thresholding based on the voxel intensity. Each nanoparticle or agglomerate of nanoparticles is shown with a color that differs from those of its nearest neighbors. Arrows indicate small nanoparticles/agglomerates that are successfully captured in the segmented CS-ET reconstruction, but are missing from the segmented SIRT reconstruction, as their reconstructed intensity is too low. 